\title{
Magnetic and Structural Properties of Organic Radicals Based on Thienyl- and Furyl-Substituted Nitronyl Nitroxide
}

\author{
Tadashi Sugano ${ }^{1, *}$, Stephen J. Blundell ${ }^{2}$, William Hayes ${ }^{2}$ and Hatsumi Mori ${ }^{3}$ \\ 1 Department of Chemistry, Meiji Gakuin University, Kamikurata, Totsuka, Yokohama 244-8539, Japan \\ 2 Clarendon Laboratory, Department of Physics, Oxford University, Parks Road, Oxford OX1 3PU, UK; \\ stephen.blundell@physics.ox.ac.uk (S.J.B.); bill.hayes@physics.ox.ac.uk (W.H.) \\ 3 The Institute for Solid State Physics, The University of Tokyo, Kashiwanoha, Kashiwa 277-8581, Japan; \\ hmori@issp.u-tokyo.ac.jp \\ * Correspondence: sugano@law.meijigakuin.ac.jp
}

Citation: Sugano, T.; Blundell, S.J.; Hayes, W.; Mori, H. Magnetic and Structural Properties of Organic Radicals Based on Thienyl- and Furyl-Substituted Nitronyl Nitroxide. Magnetochemistry 2021, 7, 62.

https://doi.org/10.3390

/magnetochemistry7050062

Academic Editors: Lee Martin, Scott Turner, John Wallis,

Hiroki Akutsu and Carlos J. Gómez García

Received: 31 March 2021

Accepted: 29 April 2021

Published: 6 May 2021

Publisher's Note: MDPI stays neutral with regard to jurisdictional claims in published maps and institutional affiliations.

Copyright: (c) 2021 by the authors. Licensee MDPI, Basel, Switzerland. This article is an open access article distributed under the terms and conditions of the Creative Commons Attribution (CC BY) license (https:/ / creativecommons.org/licenses/by/ $4.0 /)$.

\begin{abstract}
Magnetic properties of organic radicals based on thienyl- and furyl-substituted nitronyl nitroxide (NN) and iminonitroxide (IN) were investigated by measuring the temperature dependence of the magnetization. The magnetic behavior of 2-benzo[b]thienyl NN (2-BTHNN) is interpreted in terms of the two-magnetic-dimer model, in which one dimer exhibits ferromagnetic (FM) intermolecular interaction and the other dimer shows antiferromagnetic (AFM) interaction. The existence of two dimers in 2-BTHNN is supported by crystal structure analysis. The magnetic behaviors of 2-bithienyl NN, 4-(2'-thienyl)phenyl NN (2-THPNN), 2- and 3-furyl NN, 2-benzo[b]furyl NN, and 3 -benzo[b]thienyl IN are also reported. The one-dimensional alternating AFM nature observed in 2-THPNN is consistent with its crystal structure.
\end{abstract}

Keywords: nitronyl nitroxide; iminonitroxide; magnetism; organic crystals

\section{Introduction}

Magnetism in neutral organic radicals based on nitronyl nitroxide (NN) (2-substituted 4,4,5,5-tetramethyl-4,5-dihydro-3-oxido-1H-imidazol-3-ium-2-yl-1-oxyl) and iminonitroxide (IN) (2-substituted 4,4,5,5-tetramethyl-4,5-dihydro-1H-imidazol-2-yl-1-oxyl) has long been studied to find new magnetically interesting molecular crystals, since these radicals are usually stable in the solid state [1] and have been components of many molecule-based magnets [2-10].

Introducing sulfur atoms into the NN and IN derivatives would result in an increase in magnetic interactions between neighboring molecules in molecular crystals, since the sulfur atoms can make larger molecular orbital overlaps as observed in conducting organic materials [11]. We have, therefore, been preparing thienyl-substituted NN and IN, which include a sulfur atom, and investigating their magnetic properties [12-14]. We report here magnetic properties of three thienyl-substituted NN derivatives, 2-benzo[ $b$ ]thienyl NN (2-BTHNN), 2-bithienyl NN (2-BiTHNN), and 4-(2'-thenyl)phenyl NN (2-THPNN) and two thienyl-substituted IN derivatives, 2- and 3-benzo[b]thienyl IN (2-BTHIN and 3-BTHIN). We also report here crystal structures of 2-BTHNN and 2-THPNN to discuss magnetostructural correlations in these radicals. To discuss the effects of sulfur substitution on magnetic interactions, magnetic properties of three furyl-substituted NN derivatives, 2- and 3-furyl NN (2-FNN and 3-FNN), and 2-benzo[b]furyl NN (2-BFNN), and a furyl-substituted IN derivative, 2-furyl IN (2-FIN), are also described, because the furyl ring is the oxygen analogue of the thienyl ring. The molecular structures of organic radicals reported here are listed in Figure 1. 


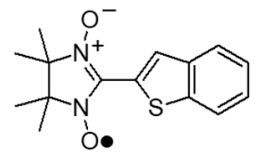

2-BTHNN

(a)

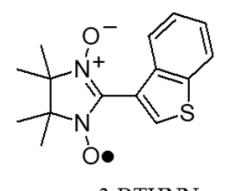

(c)

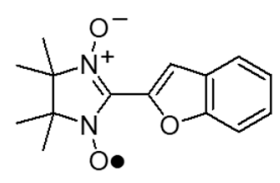

2-BFNN

(e)<smiles>CC1(C)N([O])C(c2ccco2)=[N+]([O-])C1(C)C</smiles>

2-FNN

(g)

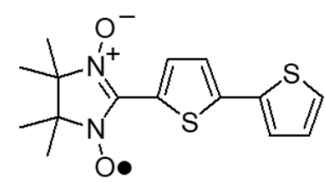

2-BiTHNN

(i)<smiles>CC1(C)N=C(c2cc3ccccc3s2)N([O-])C1(C)C</smiles>

2-BTHIN

(b)<smiles>CC1(C)N=C(c2csc3ccccc23)N(O)C1(C)C</smiles>

(d)<smiles>CC1(C)N([O])C(c2ccoc2)=[N+]([O-])C1(C)C</smiles>

3-FNN

(f)<smiles>CC1(C)N=C(c2ccco2)N([O])C1(C)C</smiles>

2-FIN

(h)

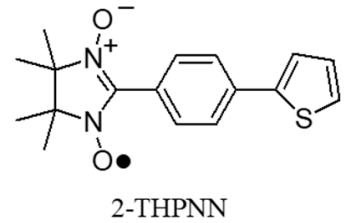

(j)

Figure 1. Molecular structures of organic radicals reported here. (a) 2-BTHNN, (b) 2-BTHIN, (c) 3BTHNN, (d) 3-BTHIN, (e) 2-BFNN, (f) 3-FNN, (g) 2-FNN, (h) 2-FIN, (i) 2-BiTHNN, and (j) 2-THPNN (see the text for the abbreviated names).

This paper is a tribute to Professor Peter Day who gave us many suggestions and opportunities to carry out our studies of magnetochemistry.

\section{Results and Discussion}

\subsection{Magnetic Properties}

Figure 2 shows the temperature dependence of the product of paramagnetic susceptibility $\chi_{p}$ and temperature T of 2-BTHNN and 2-BTHIN. Upon lowering the temperature, the product, $\chi_{p} T$, of 2-BTHNN decreases monotonically from $0.374 \mathrm{emu} \cdot \mathrm{K} \cdot \mathrm{mol}^{-1}$ at $300 \mathrm{~K}$ to $0.198 \mathrm{emu} \cdot \mathrm{K} \cdot \mathrm{mol}^{-1}$ at around $10 \mathrm{~K}$. Below about $10 \mathrm{~K}$, however, $\chi_{p} T$ of 2-BTHNN increases slowly to $0.204 \mathrm{emu} \cdot \mathrm{K} \cdot \mathrm{mol}^{-1}$ at $1.8 \mathrm{~K}$, suggesting the existence of ferromagnetic (FM) interactions in 2-BTHNN. 


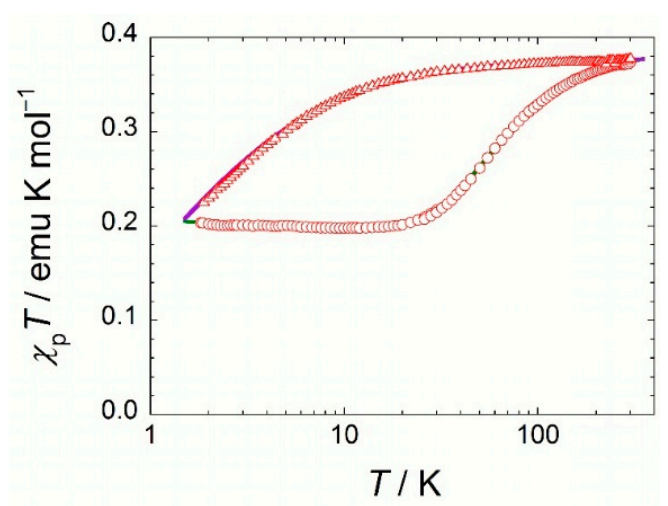

Figure 2. Temperature dependences of $\chi_{p} T$ of 2-BTHNN (open circles) and 2-BTHIN (open triangles). The solid line for 2-BTHNN indicates a fit using the two-magnetic-dimer model (see the text).

Since the temperature dependence of $\chi_{p} T$ of 2-BTHNN shown above appears not to be simple, we examined several models to fit the data and then found that the twomagnetic-dimer model, in which one molecular dimer (which we denote as the FM dimer hereafter) exhibits FM intermolecular interactions and the other dimer (which we denote as the AFM dimer) shows antiferromagnetic (AFM) intermolecular interactions, can explain the temperature dependence of $\chi_{p} T$ of 2-BTHNN, as represented by the solid line in Figure 2. At temperatures lower than about $10 \mathrm{~K}$, the moderately strong AFM interaction operating in the AFM dimer, mentioned below, leads to an almost complete vanishing of the contribution of the AFM dimer to $\chi_{p}$. This AFM interaction is interpreted in terms of the two-spin dimer model [15] with the exchange coupling constant $J / k=-55 \mathrm{~K}$ and the Curie constant $C=0.197 \mathrm{emu} \cdot \mathrm{K} \cdot \mathrm{mol}^{-1}$. This magnitude of $C$ is about a half of $0.376 \mathrm{emu} \cdot \mathrm{K} \cdot \mathrm{mol}^{-1}$ for the uncorrelated $S=1 / 2$ spins in 2-BTHNN

As a result, the temperature dependence of $\chi_{p} T$ of 2-BTHNN below about $10 \mathrm{~K}$ would come from only the contribution of the FM dimer. This contribution is modeled in terms of the Curie-Weiss law with the Weiss temperature $\theta=+0.06 \mathrm{~K}$ and $C=0.197 \mathrm{emu} \cdot \mathrm{K} \cdot \mathrm{mol}^{-1}$. The positive Weiss temperature obtained here clearly indicates the existence of the FM intermolecular interactions in the FM dimer. In addition, we observed further evidence for the FM interactions by measuring the magnetization isotherms at low temperatures below $10 \mathrm{~K}$. Upon lowering temperature, magnetization isotherms deviate from the $S=1 / 2$ Brillouin function curve onto the $S=1$ curve as shown in Figure 3. Since the Curie constant for the FM dimer is the same as that of the AFM dimer and it is just a half of the Curie constant for the uncorrelated $S=1 / 2$ spins of 2-BTHNN, it is concluded that the half of the molecular spins exhibit the FM intermolecular interactions and the other half of spins show the AFM interactions in 2-BTHNN. This conclusion is supported further by analyzing the crystal structure of 2-BTHNN as mentioned below.

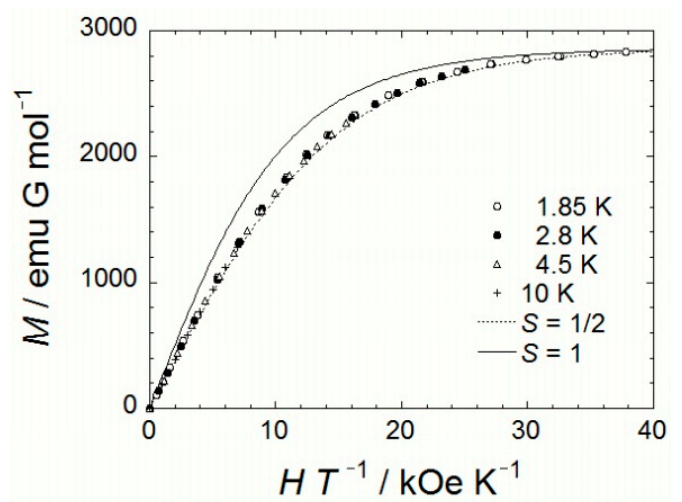

Figure 3. Magnetization isotherms of 2-BTHNN at $1.85 \mathrm{~K}$ (open circles), $2.8 \mathrm{~K}$ (closed circles), $4.5 \mathrm{~K}$ (open triangles), and $10 \mathrm{~K}$ (crosses). 
In contrast, the temperature dependences of $\chi_{p} T$ of 2-BTHIN can be fitted simply to the Curie-Weiss law with $C=0.378 \mathrm{emu} \cdot \mathrm{K} \cdot \mathrm{mol}^{-1}$ and $\theta=-1.23 \mathrm{~K}$. In this case, our results show that the elimination of an oxygen atom gives a drastic change of magnetic behavior.

Figure 4 shows the temperature dependences of $\chi_{p} T$ of 2-BiTHNN (open circles) and 2-THPNN (open triangles). These two radicals also have thienyl-including moieties that are longer than those of other radicals reported in this paper, as shown in Figure 1. These two radicals exhibit weak AFM intermolecular interactions, because the temperature dependences of $\chi_{p}$ T of 2-BiTHNN and 2-THPNN are interpreted in terms of the one-dimensional (1D) alternating Heisenberg model [16] with $\mathrm{J} / \mathrm{k}=-2.34 \mathrm{~K}$, alternating parameter $\alpha=0.8$ and $C=0.380 \mathrm{emu} \cdot \mathrm{K} \cdot \mathrm{mol}^{-1}$, and with $\mathrm{J} / \mathrm{k}=-0.77 \mathrm{~K}, \alpha=0.8$, and $C=0.370 \mathrm{emu} \cdot \mathrm{K} \cdot \mathrm{mol}^{-1}$, respectively, as represented by the solid lines in Figure 4 . The origin of the alternating magnetic interactions in 2-THPNN is discussed below by referring to the crystal structure.

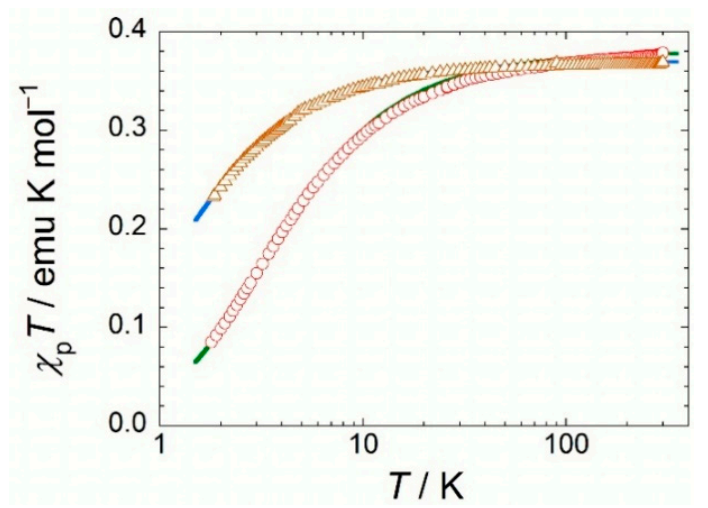

Figure 4. Temperature dependences of $\chi_{p} T$ of 2-BiTHNN (open circles) and 2-THPNN (open triangles). Solid lines represent theoretical fitting on the basis of the 1D alternating AFM Heisenberg model (see the text).

The magnetic properties of 4-(3'-thienyl)phenyl NN (3-THPNN), which is a structural isomer of 2-THPNN shown here, was previously reported by Coronado et al. about two decades ago [17]. The radical 3-THPNN shows weak AFM intermolecular interactions with $\theta=-1.5 \mathrm{~K}$ and $C=0.31 \mathrm{emu} \cdot \mathrm{K} \cdot \mathrm{mol}^{-1}$ similar to those found in 2-THPNN.

The temperature dependences of $\chi_{p} T$ of the three furyl-substituted nitronyl nitroxide radicals, 2-FNN (open circles), 3-FNN (open triangles), and 2-BFNN (open squares), are shown in Figure 5 together with those of furyl-substituted iminonitroxide radical, 2-FIN. The magnetic behaviors of 3-FNN, 2-BFNN, and 2-FIN are similar to each other, although the magnitude of the magnetic interaction is significantly different as mentioned below, while those of 2-FNN are quite different at temperatures lower than about $10 \mathrm{~K}$. The values of $\chi_{p} T$ do not decrease steeply with lowering temperature but show a plateau between 4 and $10 \mathrm{~K}$. Although this behavior appears to be reminiscent of that observed in 2-BTHNN, a similar kind of behavior is also characteristic of the four-spin linear tetramer model [18], because any upturn of $\chi_{p} T$ values at low temperatures is not observed. As represented by the solid line in Figure 5, we successfully reproduced the temperature dependence of $\chi_{p} T$ of 2 -FNN in terms of the four-spin linear tetramer model with $J_{1} / k=-3.5 \mathrm{~K}$ and $J_{2} / k=-13 \mathrm{~K}$, where $J_{1} / k$ represents the intradimer interaction and $J_{2} / k$ represents the interdimer interaction in the linear tetramer, having $S=1 / 2$ spin on each molecule within the tetramer. The Curie constant used to fit the experimental data was $C=0.340 \mathrm{emu} \cdot \mathrm{K} \cdot \mathrm{mol}^{-1}$. This value is slightly lower than the value $C=0.376 \mathrm{emu} \cdot \mathrm{K} \cdot \mathrm{mol}^{-1}$ that is expected for uncorrelated $S=1 / 2$ spins. This difference comes from the contribution of impurity spins with $C_{i}=0.038 \mathrm{emu} \cdot \mathrm{K} \cdot \mathrm{mol}^{-1}$ and $\theta_{i}=-0.1 \mathrm{~K}$ used to obtain the best fit to the experimental data. The overall Curie constant is $0.378 \mathrm{emu} \cdot \mathrm{K} \cdot \mathrm{mol}^{-1}$ and close to that expected for uncorrelated $S=1 / 2$ spins. The origin of spin interactions within the tetramer is not clear, since we have no crystal information for 2-FNN at present. 


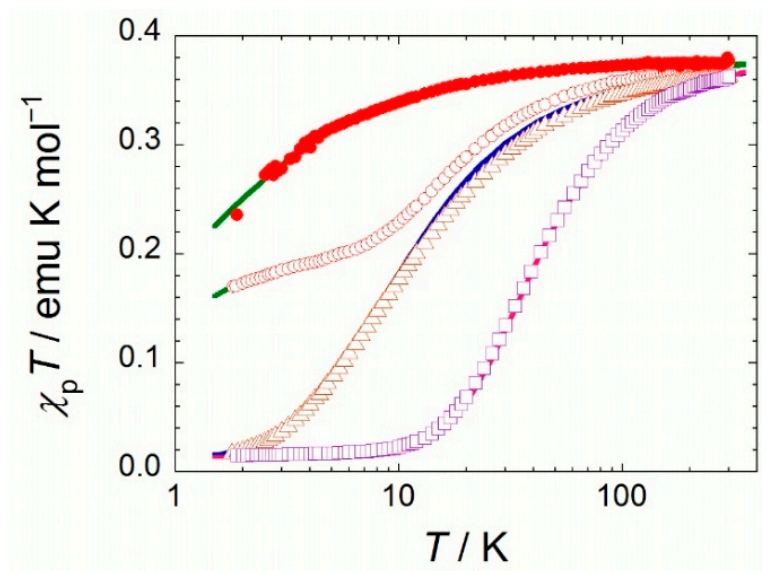

Figure 5. Temperature dependences of $\chi_{p} T$ of 2-FNN (open circles), 3-FNN (open triangles), 2-BFNN (open squares), and 2-FIN (closed circles). The solid lines represent fits using the four-spin linear tetramer model for 2-FNN, the 1D alternating AFM Heisenberg model for 3-FNN and 2-BFNN, and the Curie-Weiss law for 2-FIN (see the text).

The temperature dependences of $\chi_{p} T$ of 3-FNN and 2-BFNN can be fitted to the 1D alternating Heisenberg model [16] with $J / k=-7.5 \mathrm{~K}, \alpha=0.6, C=0.346 \mathrm{emu} \cdot \mathrm{K} \cdot \mathrm{mol}^{-1}$, $C_{i}=0.025 \mathrm{emu} \cdot \mathrm{K} \cdot \mathrm{mol}^{-1}$ and $\theta_{i}=-0.0 \mathrm{~K}$ for $3-\mathrm{FNN}$, and with $J / k=-33 \mathrm{~K}, \alpha=0.2, C=0.369 \mathrm{emu} \cdot \mathrm{K} \cdot \mathrm{mol}^{-1}$, $C_{i}=0.022 \mathrm{emu} \cdot \mathrm{K} \cdot \mathrm{mol}^{-1}, \theta_{i}=-1.0 \mathrm{~K}$ for $2-\mathrm{BFNN}$. On the other hand, the temperature dependence of $\chi_{p} T$ of 2-FIN interpreted in terms of the Curie-Weiss law yields $C=0.376 \mathrm{emu} \cdot \mathrm{K} \cdot \mathrm{mol}^{-1}$ and $\theta=-1.0 \mathrm{~K}$.

The sulfur analogues of 2- and 3-FNN, 2- and 3-THNN, show 1D alternating Heisenberg behavior with $J / k=-6.6 \mathrm{~K}, \alpha=0.5, C=0.359 \mathrm{emu} \cdot \mathrm{K} \cdot \mathrm{mol}^{-1}, C_{i}=0.009 \mathrm{emu} \cdot \mathrm{K} \cdot \mathrm{mol}^{-1}$, $\theta_{i}=0.0 \mathrm{~K}$ for $2-\mathrm{THNN}$, and with $\mathrm{J} / \mathrm{k}=-5.3 \mathrm{~K}, \alpha=0.6, \mathrm{C}=0.360 \mathrm{emu} \cdot \mathrm{K} \cdot \mathrm{mol}^{-1}, C_{i}=0.016 \mathrm{emu} \cdot \mathrm{K} \cdot \mathrm{mol}^{-1}$, $\theta_{i}=0.0 \mathrm{~K}$ for 3-THNN [14]. The magnetic behavior of 2-FNN mentioned above is very different from that of 2-THNN. That is to say, 2-FNN shows the four-spin linear tetramer behavior and 2-THNN exhibits 1D alternating Heisenberg behavior. It is, therefore, difficult to compare magnetic interactions directly in both radicals. However, the magnetic behaviors of 3-FNN and 3-THNN are both interpreted in terms of the 1D alternating Heisenberg model with $J / k=-7.5 \mathrm{~K}(\alpha=0.6)$ and $J / k=-5.3 \mathrm{~K}(\alpha=0.6)$, respectively. This result suggests that the substitution of the oxygen atom in the furyl ring by the sulfur atom does not yield stronger magnetic interactions in this case.

The magnetic interactions in 2-BTHNN, which is the sulfur analogue of 2-BFNN, seem to become stronger. The magnetic behavior of 2-BFNN can also be explained by using the two-spin dimer model with $J / k=-35 \mathrm{~K}$, although the $1 \mathrm{D}$ alternating Heisenberg model with $J / k=-34 \mathrm{~K}(\alpha=0.2)$ gives slightly better fit as mentioned above. The value of $J / k=-35 \mathrm{~K}$ is smaller than $J / k=-55 \mathrm{~K}$ as observed in 2-BTHNN. This result indicates that the substitution of the oxygen atom in the furyl ring by the sulfur atom yields stronger magnetic interactions in this case.

The temperature dependences of $\chi_{p} T$ of 3-BTHIN is shown in Figure 6 together with that of 3-BTHNN [14]. The magnetic behavior of 3-BTHIN is reproduced in terms of the 1D AFM Heisenberg model with $J / k=-1.78 \mathrm{~K}, C=0.348 \mathrm{emu} \cdot \mathrm{K} \cdot \mathrm{mol}^{-1}, C_{i}=0.032 \mathrm{emu} \cdot \mathrm{K} \cdot \mathrm{mol}^{-1}$ and $\theta_{i}=0.0 \mathrm{~K}$, whereas that of 3-BTHNN is interpreted in terms of quasi-two-dimensional FM intermolecular interactions with $J / k=+0.16 \mathrm{~K}$ within the layer and $J^{\prime} / k=+0.02 \mathrm{~K}$ for the interlayer and $C=0.384 \mathrm{emu} \cdot \mathrm{K} \cdot \mathrm{mol}^{-1}$ [14]. Elimination of an oxygen atom from one of the NO groups of the nitronyl nitroxide moiety of 3-BTHNN results in a remarkable change in magnetic behavior due possibly to a change in molecular arrangements in the solid. To discuss further, it is indispensable to determine the crystal structures of 3-BTHIN. 


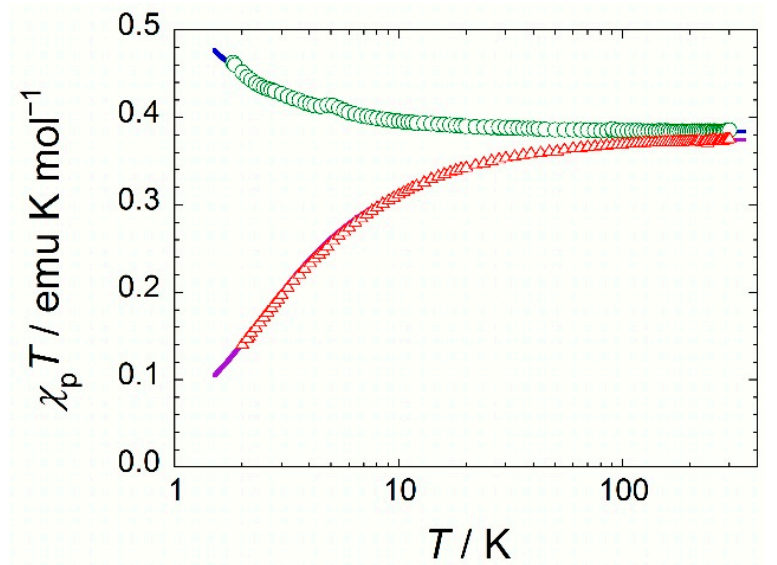

Figure 6. Temperature dependences of $\chi_{p} T$ of 3-BTHIN (open triangles) and 3-BTHNN (open circles). Solid lines represent theoretical fitting on the basis of the 1D AFM Heisenberg model for 3-BTHIN and the quasi-2D FM Heisenberg model for 3-BTHNN (see the text).

\subsection{Crystal Structures}

The radical 2-BTHNN crystallizes in the monoclinic space group C2/c. The crystallographic data of 2-BTHNN are listed in Table 1. Figure 7a shows an ORTEP view of the crystal structure along the $b$ axis. The 2-BTHNN molecules form two different types of molecular dimers as denoted by A and B shown in Figure 7a. In dimer A, the 2-BTHNN molecules stack face-to-face and their molecular long axes make an angle of $73^{\circ}$, as shown in Figure $7 \mathrm{~b}$. The shortest intermolecular atomic distance between $\mathrm{N}$ and $\mathrm{O}$ atoms is 3.499(3) $\AA$. In dimer B, the 2-BTHNN molecules stack face-to-face in a head-to-tail manner as shown in Figure 7c. The benzothienyl rings are close to each other to avoid steric hindrances due to bulky methyl groups on the nitronyl nitroxide moieties. The shortest intermolecular atomic distance is 3.267(5) $\AA$ between the $C$ atom of the benzene ring and the $C$ atom of the thiophene ring. Quite different molecular arrangements in these two dimers $\mathrm{A}$ and $\mathrm{B}$ mentioned above would yield very distinctive magnetic behaviors, i.e., FM and AFM interactions in 2-BTHNN. Although it is not easy to attribute the origin of FM and AFM interactions onto these different dimers, the nearly orthogonally arranged molecules in the dimer A seems to give the FM interactions, and the face-to-face stacking of benzothienyl groups appears to result in the AFM interactions in 2-BTHNN.

Table 1. Crystallographic data for the organic radicals 2-BTHNN and 2-THPNN.

\begin{tabular}{ccc}
\hline & 2-BTHNN & 2-THPNN \\
\hline Chemical formula & $\mathrm{C}_{15} \mathrm{H}_{17} \mathrm{~N}_{2} \mathrm{O}_{2} \mathrm{~S}$ & $\mathrm{C}_{17} \mathrm{H}_{19} \mathrm{~N}_{2} \mathrm{O}_{2} \mathrm{~S}$ \\
Formula weight & 289.37 & 315.41 \\
Crystal system & Monoclinic & Monoclinic \\
Space group & $\mathrm{C} 2 / \mathrm{c}$ & $\mathrm{P}_{1} / \mathrm{n}$ \\
$a(\AA)$ & $17.636(6)$ & $13.30(7)$ \\
$b(\AA)$ & $11.400(4)$ & $9.57(4)$ \\
$c(\AA)$ & $29.886(9)$ & $14.51(7)$ \\
$\beta\left({ }^{\circ}\right)$ & $95.442(4)$ & $117.28(7)$ \\
$V\left(\AA^{3}\right)$ & $5982(3)$ & $1642(14)$ \\
$Z$ value & 16 & 4 \\
Dcalc $\left(\mathrm{Mg} \cdot \mathrm{m}^{-3}\right)$ & 1.285 & 1.276 \\
Reflections independent & 6259 & 3631 \\
$R, R_{w}[I>2 \sigma(\mathrm{I})]$ & $0.0808,0.1002$ & $0.0808,0.0967$
\end{tabular}


(a)

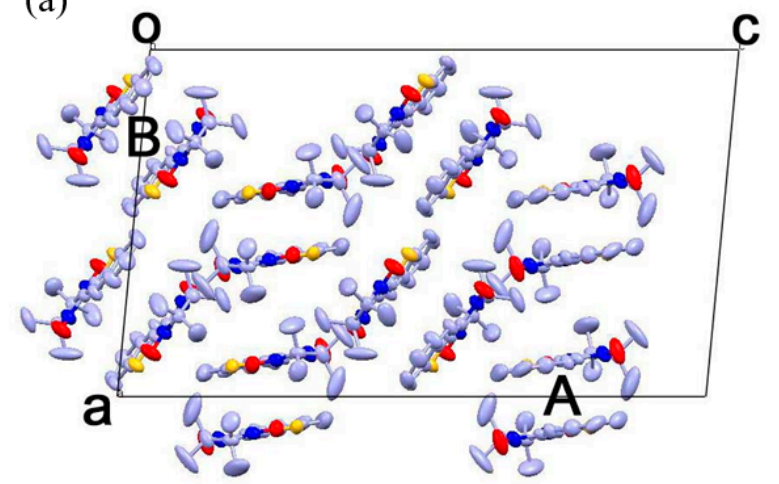

(b)

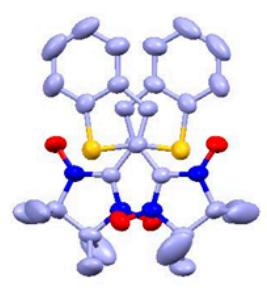

(c)

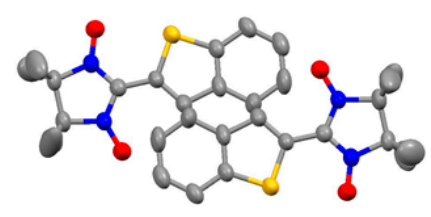

Figure 7. (a) An ORTEP view of the crystal structure of 2-BTHNN along the $b$ axis. "A" and "B" denote the two kinds of molecular dimers. Molecular conformations of two neighboring 2-BTHNN in dimer A (b) and dimer B (c).

The radical 2-THPNN crystallizes in the monoclinic space group $\mathrm{P} 2{ }_{1} / \mathrm{n}$. The crystallographic data of 2-THPNN are also listed in Table 1. Figure 8 shows an ORTEP view of the crystal structure along the direction perpendicular to the molecular planes of the 2-THPNN within one of the molecular stacks. The 2-THPNN molecules stack side-by-side in a head-to-tail manner along the $a$ axis. The molecular planes of the molecules belong to the neighboring stacks are arranged perpendicularly. In the molecular stacks, there are two types of atomic contacts between neighboring molecules. One type of atomic contact is formed between the $\mathrm{O}$ atom on the $\mathrm{NO}$ group and the two $\mathrm{C}$ atoms on the phenyl ring with the atomic distances of 3.404(2) $\AA$ and 3.449(3) $\AA$. The other type of atomic contact is formed between the $\mathrm{O}$ atom and the $\mathrm{C}$ atom on the phenyl ring with the atomic distance of 3.309(3) $\AA$ and the $C$ atom on the thienyl ring with the atomic distance of 3.462(3) $\AA$. These two types of atomic contacts existing in the molecular stacks along the $a$ axis probably result in the 1D alternating magnetic interactions observed in 2-THPNN.

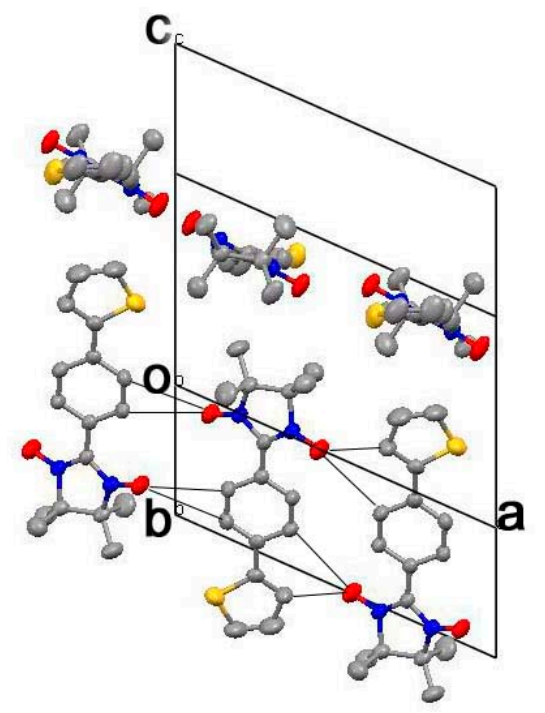

Figure 8. An ORTEP view of the crystal structure of 2-THPNN along the vertical direction to the molecular planes. The 2-THPNN molecules form side-by-side molecular stacks along the $a$ axis in a head-to-tail manner.

\section{Materials and Methods}

The radicals were prepared according to the procedures reported in [1] and purified through column chromatography followed by a recrystallization. Commercially available (Aldrich) benzo[ $b]$ thiophene-2-carboxyaldehyde, benzo[ $b]$ thiophene-3-carboxyaldehyde, 2-benzofurancarboxyaldehyde, 2-furanaldehyde, 3-furanaldehyde, 2,2'-bithio-phene-5- 
carboxyaldehyde, and 4-(2'-thienyl)benzaldehyde were used without further purification. $N, N^{\prime}$-Dihydroxy-2,3-diamino-2,3-dimethylbutane was obtained according to the literature [1]. Other reagents and solvents were used as purchased.

2-BTHNN and 2-BTHIN. Benzo[b]thiophene-2-carboxyaldehyde (2.56 g, $15.8 \mathrm{mmol})$ and $N, N^{\prime}$-dihydroxy-2,3-diamino-2,3-dimethylbutane $(2.14 \mathrm{~g}, 14.4 \mathrm{mmol})$ were mixed in $15 \mathrm{~mL}$ of benzene at $40{ }^{\circ} \mathrm{C}$. The reaction mixture was stirred for $24 \mathrm{~h}$, after which the resulting white solid of 1,3-dihydroxy-2-(2-bebzo[b]thienyl)-4,4,5,5-tetramethylimidazolidine was filtered off and washed with $5 \mathrm{~mL}$ benzene twice and dried under vacuum. Yield: $98 \%$. A solution of sodium periodate $(2.42 \mathrm{~g}, 11.3 \mathrm{mmol})$ in $25 \mathrm{~mL}$ of water was added dropwise to a suspension of 1,3-dihydroxy-2-(2-bebzo[ $b$ ]thienyl)-4,4,5,5-tetramethylimidazolidine $(2.18 \mathrm{~g}, 7.47 \mathrm{mmol})$ in $100 \mathrm{~mL}$ dichloromethane at room temperature. The dark-green organic phase was separated and concentrated under vacuum. The crude product was separated and purified by column chromatography (eluent: ethyl acetate, alumina) to obtain 2-BTHNN (dark-green solid) and 2-BTHIN (red solid).

3-BTHNN and 3-BTHIN. A similar experimental procedure was used to obtain 3BTHNN (dark-green/blue solid) and 3-BTHIN (red solid) by using benzo[b]thiophene-3carboxyaldehyde.

2-BFNN. 2-Benzofurancarboxyaldehyde $(2.58 \mathrm{~g}, 17.7 \mathrm{mmol})$ and $N, N^{\prime}$-dihydroxy2,3-diamino-2,3-dimethylbutane $(2.39 \mathrm{~g}, 16.1 \mathrm{mmol})$ were mixed in $15 \mathrm{~mL}$ of benzene at $40{ }^{\circ} \mathrm{C}$. The reaction mixture was stirred for $2 \mathrm{~h}$, after which the resulting white solid of 1,3-dihydroxy-2-(2-bebzo[b]furyl)-4,4,5,5-tetramethylimidazolidine was filtered off and washed with $5 \mathrm{~mL}$ of benzene twice and dried under vacuum. Yield: $95 \%$. A solution of sodium periodate $(2.74 \mathrm{~g}, 12.8 \mathrm{mmol})$ in $25 \mathrm{~mL}$ of water was added dropwise to a suspension of 1,3-dihydroxy-2-(2-bebzo[b]furyl)-4,4,5,5-tetramethylimidazolidine (2.30 g, $8.33 \mathrm{mmol}$ ) in $100 \mathrm{~mL}$ of dichloromethane at room temperature. The dark-green organic phase was separated and concentrated under vacuum. The crude product was separated and purified by column chromatography (eluent: ethyl acetate, alumina) to obtain 2-BFNN (dark-green solid).

3-FNN. 3-Furanaldehyde (1.20 g, $12.4 \mathrm{mmol})$ and $N, N^{\prime}$-dihydroxy-2,3-diamino-2,3dimethylbutane $(1.71 \mathrm{~g}, 11.5 \mathrm{mmol})$ were mixed in $10 \mathrm{~mL}$ of benzene at $40^{\circ} \mathrm{C}$. The reaction mixture was stirred for $24 \mathrm{~h}$, after which the resulting light-brown solid of 1,3-dihydroxy2-(3-furyl)-4,4,5,5-tetramethylimidazolidine was filtered off and washed with $5 \mathrm{~mL}$ of benzene twice and dried under vacuum. Yield: $75 \%$. A solution of sodium periodate $(2.80 \mathrm{~g}$, $13.1 \mathrm{mmol})$ in $25 \mathrm{~mL}$ of water was added dropwise to a suspension of 1,3-dihydroxy-2-(3furyl)-4,4,5,5-tetramethylimidazolidine (1.95 g, $8.63 \mathrm{mmol})$ in $100 \mathrm{~mL}$ of dichloromethane at room temperature. The dark-blue organic phase was separated and concentrated under vacuum. The crude product was separated and purified by column chromatography (eluent: ethyl acetate, alumina) to obtain 3-FNN (dark-blue solid). In this case, enough amount of 3-FIN was not obtained as a byproduct.

2-FNN and 2-FIN. 2-Furanaldehyde $(1.93 \mathrm{~g}, 20.1 \mathrm{mmol})$ and $N, N^{\prime}$-dihydroxy-2,3diamino-2,3-imethylbutane $(2.71 \mathrm{~g}, 18.3 \mathrm{mmol})$ were mixed in $10 \mathrm{~mL}$ of benzene at $40{ }^{\circ} \mathrm{C}$. The reaction mixture was stirred for $20 \mathrm{~h}$ after which the resulting light-brown solid of 1,3-dihydroxy-2-(2-furyl)-4,4,5,5-tetramethylimidazolidine was filtered off and washed with $5 \mathrm{~mL}$ benzene twice and dried under vacuum. Yield: 74\%. A solution of sodium periodate $(2.14 \mathrm{~g}, 10.0 \mathrm{mmol})$ in $20 \mathrm{~mL}$ of water was added dropwise to a suspension of 1,3-dihydroxy-2-(2-furyl)-4,4,5,5-tetramethylimidazolidine (1.50 g, $6.64 \mathrm{mmol})$ in $100 \mathrm{~mL}$ of dichloromethane at room temperature. The dark-blue organic phase was separated and concentrated under vacuum. The crude product was separated and purified by column chromatography (eluent: ethyl acetate, alumina) to obtain 2-FNN (dark-blue solid) and 2-FIN (red solid).

2-BiTHNN. 2,2'-bithiophene-5-carboxyaldehyde (1.56 g, $7.98 \mathrm{mmol})$ and $N, N^{\prime}$-dihydroxy2,3-diamino-2,3-imethylbutane $(1.10 \mathrm{~g}, 7.39 \mathrm{mmol})$ were mixed in $10 \mathrm{~mL}$ of benzene at $40{ }^{\circ} \mathrm{C}$. The reaction mixture was stirred for $20 \mathrm{~h}$, after which the resulting light-yellow /brown solid of 1,3-dihydroxy-2-(2'-bithienyl)-4,4,5,5-tetramethylimidazolidine was filtered off and 
washed with $3 \mathrm{~mL}$ of benzene five times and dried under vacuum. Yield: $63 \%$. A solution of sodium periodate $(1.50 \mathrm{~g}, 6.98 \mathrm{mmol})$ in $15 \mathrm{~mL}$ of water was added dropwise to a suspension of 1,3-dihydroxy-2-(2'-bithienyl)-4,4,5,5-tetramethylimidazolidine (1.50 g, $4.62 \mathrm{mmol})$ in $100 \mathrm{~mL}$ of dichloromethane at room temperature. The dark-green organic phase was separated and concentrated under vacuum. The crude product was separated and purified by column chromatography (eluent: ethyl acetate, alumina) to obtain 2-BiTHNN (dark-green solid).

2-THPNN. 4-(2'-thienyl)benzaldehyde (1.00 g, $5.32 \mathrm{mmol})$ and $N, N^{\prime}$-dihydroxy-2,3diamino-2,3-imethylbutane $(0.726 \mathrm{~g}, 4.90 \mathrm{mmol})$ were mixed in $10 \mathrm{~mL}$ of benzene at $40{ }^{\circ} \mathrm{C}$. The reaction mixture was stirred for $24 \mathrm{~h}$, after which the resulting light-brown solid of 1,3-dihydroxy-2-[4-(2'-thienyl)phenyl]-4,4,5,5-tetramethylimidazolidine was filtered off and washed with $5 \mathrm{~mL}$ of benzene three times and dried under vacuum. Yield: 88\%. A solution of sodium periodate $(1.39 \mathrm{~g}, 6.51 \mathrm{mmol})$ in $15 \mathrm{~mL}$ of water was added dropwise to a suspension of 1,3-dihydroxy-2-[4-(2'-thienyl)phenyl]-4,4,5,5-tetramethylimidazolidine $(1.38 \mathrm{~g}, 4.32 \mathrm{mmol})$ in $80 \mathrm{~mL}$ of dichloromethane at room temperature. The dark-green organic phase was separated and concentrated under vacuum. The crude product was separated and purified by column chromatography (eluent: ethyl acetate, alumina) to obtain 2-THPNN (dark-green solid).

Crystals suitable for X-ray diffraction studies were grown by slow evaporation from concentrated solutions of 2-BTHNN and 2-THPNN in toluene in the dark and cold room.

The magnetization isotherms up to $7 \mathrm{~T}$ and the magnetic susceptibility over the temperature range from $1.8 \mathrm{~K}$ to $300 \mathrm{~K}$ were measured using Quantum Design MPMSXL7 SQUID (superconducting quantum interference device) magnetometers. The contribution of the diamagnetism to the susceptibility was subtracted by extrapolating the temperature dependence of the susceptibility to high temperatures where the Curie-Weiss law is applicable.

X-ray diffraction intensities were recorded on a Rigaku AFC10 automatic four-circle diffractometer with graphite monochromated Mo-K $\alpha(\lambda=71.075 \mathrm{pm})$. Intensity data were corrected for Lorentz and polarization effects but not for absorption. The crystal structures were solved by the direct methods and the positions of hydrogen atoms were calculated. A full-matrix least-square refinement was carried out, in which non-hydrogen atoms were treated with anisotropic thermal parameters and those of hydrogen atoms were treated isotropic parameters. The X-ray crystallographic CIF files for 2-BTHNN and 2-TPHNN are available as CCDC2079877 and CCDC2079881, respectively.

\section{Conclusions}

We showed magneto-structural correlations in the radicals 2-BTHNN and 2-THPNN by considering the results of magnetic measurements and X-ray crystallographic analyses. The coexistence of the FM and AFM intermolecular interactions in 2-BTHNN arises from the formation of two different types of radical molecular dimers. The 1D alternating AFM intermolecular interactions in 2-THPNN come from the molecular arrangements of chainlike side-by-side and head-to-tail stacking. We discussed the atomic substitution effects on magnetism by comparing the magnetic behaviors of thienyl- and furyl-substituted nitronyl nitroxide. We also investigated the effects of $\mathrm{O}$ atom elimination from the $\mathrm{NO}$ group on magnetism by comparing the magnetic behaviors of nitronyl nitroxide and iminonitroxide having the same attached moieties.

Author Contributions: Writing—original draft preparation, T.S.; writing—review and editing, S.J.B.; H.M.; supervision, W.H. All authors have read and agreed to the published version of the manuscript.

Funding: This research received no external funding.

Conflicts of Interest: The authors declare no conflict of interest. 


\section{References}

1. Ullman, E.F.; Osiecki, J.H.; Boocock, D.G.B.; Darcy, R. Studies of stable free radicals. X. Nitronyl nitroxide monoradicals and biradicals as possible small molecule spin labels. J. Am. Chem. Soc. 1972, 94, 7049-7059. [CrossRef]

2. Awaga, K.; Maruyama, Y. Ferromagnetic intermolecular interaction of the organic radical, 2-(4-nitrophenyl)-4,4,5,5-tetramethyl4,5-dihydro-1H-imidazolyl-1-oxy-3-oxide. Chem. Phys. Lett. 1989, 158, 556-558. [CrossRef]

3. Kinoshita, M.; Turek, P.; Tamura, M.; Nozawa, K.; Shiomi, D.; Nakazawa, Y.; Ishikawa, M.; Takahashi, M.; Awaga, K.; Inabe, T.; et al. An organic radical ferromagnet. Chem. Lett. 1991, 20, 1225-1228. [CrossRef]

4. Awaga, K.; Inabe, T.; Maruyama, Y. Ferromagnetic intermolecular interaction and crystal structure of the $p$-pyridyl nitronyl nitroxiide radical. Chem. Phys. Lett. 1992, 190, 349-352. [CrossRef]

5. Nakazawa, Y.; Tamura, M.; Shirakawa, N.; Shiomi, D.; Takahashi, M.; Kinoshita, M.; Ishikawa, M. Low-temperature magnetic properties of the ferromagnetic organic radical, $p$-nitrophenyl nitronyl nitroxide. Phys. Rev. B 1992, 46, 8906-8914. [CrossRef] [PubMed]

6. Sugano, T.; Pratt, F.L.; Kurmoo, M.; Takeda, N.; Ishikawa, M.; Blundell, S.J.; Pattenden, P.A.; Valladares, R.M.; Hayes, W.; Day, P. Magnetic ordering in some organic molecular magnets. Synth. Met. 1995, 71, 1827-1828. [CrossRef]

7. Sugano, T.; Kurmoo, M.; Uekusa, H.; Ohashi, Y.; Day, P. Magneto-structural correlation in two isomeric series of nitronyl nitroxide molecular magnets: Intermolecular interactions relevant to ferromagnetic exchange in naphthl and quinolyl derivatives. J. Solid State Chem. 1999, 145, 427-442. [CrossRef]

8. Catala, L.; Feher, R.; Amabilino, D.B.; Wurst, K.; Veciana, J. Pyrazol-4-yl-substituted $\alpha$-nitronyl and $\alpha$-imino nitroxide radicals in solution and solid states. Polyhedron 2001, 20, 1563-1569. [CrossRef]

9. Sugano, T.; Blundell, S.J.; Lancaster, T.; Pratt, F.L.; Mori, H. Magnetic order in the purely organic quasi-one-dimensional ferromagnet 2-benzimidazolyl nitronyl nitroxide. Phys. Rev. B 2010, 82, 180401. [CrossRef]

10. Adriano, C.; Freitas, R.S.; Paduan-Filho, A.; Pagliuso, P.G.; Oliveira, N.F.; Lahti, P.M. Magnetic phase diagram of the organic antiferromagnet F4BImNN. Polyhedron 2017, 136, 2-4. [CrossRef]

11. Mori, T.; Kobayashi, A.; Sasaki, Y.; Kobayashi, H.; Saito, G.; Inokuchi, H. The intermolecular interaction of tetrathiafulvalene and bis(ethylenedithio)tetrathiafulvalene in organic metals. Calculation of overlaps and models of energy-band structures. Bull. Chem. Soc. Jpn. 1984, 57, 627-633. [CrossRef]

12. Sugano, T. Magnetic phase transition between ferromagnetic high-temperature phase and antiferromagnetic low-temperature phase in 5-carboxy-2-thienyl nitronyl nitroxide. Chem. Lett. 2001, 29, 32-33. [CrossRef]

13. Sugano, T. Magnetic phase transitions in organic radical crystals studied by electron spin resonance. Synth. Met. 2003, 137, 1167-1168. [CrossRef]

14. Sugano, T.; Blundel, S.J.; Hayes, W.; Day, P.; Mori, H. Magnetic and structural properties of monoradicals and diradicals based on thienyl-substituted nitronyl nitroxide. Physica B 2010, 405, S327-S330. [CrossRef]

15. Bleaney, B.; Bowers, K.D. Anomalous paramagnetism of copper acetate. Proc. R. Soc. A 1952, 214, 451-465. [CrossRef]

16. Bonner, J.C.; Fisher, M.E. Linear magnetic chains with anisotropic coupling. Phys. Rev. 1964, 135, A640-A658. [CrossRef]

17. Coronado, E.; Giménez-Saiz, C.; Nicolas, M.; Romero, F.M.; Rusanov, E.; Stoeckli-Evans, H. Synthesis, crystal structures and electronic properties of imidazoline nitroxide radicals bearing active groups in electropolymerisation. New J. Chem. 2003, 27, 490-497. [CrossRef]

18. Rubenacker, G.V.; Drumheller, J.E.; Emerson, K.; Willett, R.D. Magnetic susceptibility of $\left((\mathrm{CH})_{3} \mathrm{NH}_{2}\right)_{2} \mathrm{Cu}_{4} \mathrm{Br}_{10}, \mathrm{Chains}$ of stacked linear tetramers. J. Magn. Magn. Mater. 1986, 54-57, 1483-1484. [CrossRef] 